\title{
Fontes de Lipídeos e Monensina na Alimentação de Novilhos Nelore e sua Relação com a População de Protozoários Ciliados do Rúmen' ${ }^{1}$
}

\section{Amaury Camilo Valinote ${ }^{2}$, José Carlos Machado Nogueira Filho ${ }^{3}$, Paulo Roberto Leme ${ }^{3}$, Saulo da Luz e Silva ${ }^{4}$, José Aparecido Cunha ${ }^{5}$}

\begin{abstract}
RESUMO - Quatro novilhos Nelore, fistulados e canulados no rúmen, foram distribuídos em um delineamento quadrado latino 4 x 4 , para avaliar o caroço de algodão e o sal de cálcio de ácidos graxos como fontes de gordura assim como o efeito da monensina em dietas com caroço de algodão, sobre a população de protozoários ciliados e o pH do rúmen. Os tratamentos experimentais foram: dieta controle (CTRL), dieta com sal de cálcio de ácidos graxos (SC), dieta com caroço de algodão (CA) e dieta com caroço de algodão sem monensina (CASM). Amostras do conteúdo ruminal foram colhidas às 0, 2, 4, 6 e 8 horas após a alimentação. Foi utilizada câmara de contagem de Sedgwick-Rafter com capacidade de $1 \mathrm{~mL}$ e microscópio ótico comum provido de retículo com área de $0,4362 \mathrm{~mm}^{2}$, para identificação dos gêneros de ciliados. Não houve diferença entre os tratamentos para o $\mathrm{pH}$ do rúmen. Os tratamentos com caroço de algodão reduziram todos os gêneros de protozoários ciliados, em especial de Entodinium, provavelmente pela gordura liberada. Os protozoários dos gêneros Diplodinium, Epidinium e Eudiplodinum e o número total de protozoários foram os únicos que sofreram influência do tempo de alimentação. Sais de cálcio de ácidos graxos foram suficientemente inertes no ambiente ruminal, não afetando o número de protozoários ciliados no rúmen de bovinos Nelore. A utilização de monensina em dietas contendo caroço de algodão e alto concentrado não influenciou o número de protozoários ciliados no rúmen de bovinos Nelore.
\end{abstract}

Palavras-chave: bovinos, caroço de algodão, ionóforo, protozoologia, sais de cálcio de ácidos graxos

\section{Effects of Feeding Monensin and Different Sources of Fat on the Ruminal Population of Ciliate Protozoa in Nellore Steers}

\begin{abstract}
Four ruminally and duodenally cannulated Nellore steers were assigned to a 4 x 4 latin square to study the effects of feeding monensin $(\mathrm{M})$ and different sources of fat on the ruminal population of ciliate protozoa and ruminal $\mathrm{pH}$. The experimental diets contained (DM basis) 19\% of sugar cane and were supplemented with M [control (CTRL)], M plus calcium salts of fatty acids (CSFA), M plus whole cottonseed (MWCS), or only WCS. Ruminal contents were sampled at $0,2,4,6$, and 8 hours post-feeding. In order to identify the ciliate protozoa a Sedgwick-Rafter count cell and an optic microscope with slide area of $0.4362 \mathrm{~mm}^{2}$ were used. No significant differences in ruminal $\mathrm{pH}$ were observed among diets. Both cottonseed treatments (MWCS and WCS) reduced the ruminal population of the ciliate protozoa investigated, mainly the Entodinium, possibly because of the slow release of fat. Ruminal counts of Diplodinium, Epidinium, Eudiplodinium, and total protozoa were all affected by time post-feeding. Calcium salts of fatty acids seemed to be inert in the ruminal environment because no significant changes were observed in the protozoa counts. Moreover, feeding CRTL and MWCS diets to Nellore steers did not change the ruminal population of ciliate protozoa in this trial.
\end{abstract}

Key Words: calcium salt of fatty acid, cattle, cottonseed, ionophores, protozoology

\section{Introdução}

Gorduras e óleos têm sido utilizados na alimentação de ruminantes em substituição a altas proporções de grãos, com o intuito de aumentar a densidade energética da dieta, aumentando a eficiência alimentar, além de garantir a ingestão de fibra necessária para o bom funcionamento do rúmen. Substituindo altos teores de grãos, os lipídeos evitam transtornos causados pela alta ingestão de amido (Chalupa et al., 1984; Palmquist et al., 1993).

A adição de lipídeos na ração em níveis superiores a $7 \%$ da matéria seca pode prejudicar a degradação do alimento. Os lipídeos presentes na maioria dos alimentos utilizados na alimentação animal possuem em sua constituição maiores proporções de ácidos graxos insaturados (Van Soest, 1994), que são tóxicos aos microrganismos ruminais, principalmente às bac-

\footnotetext{
${ }_{1}^{1}$ Projeto financiado pela Fapesp, Proc. 02/08632-1R

2 Doutorando da Faculdade de Zootecnia e Engenharia de Alimentos da Universidade de São Paulo, Departamento de Zootecnia. Rua Duque de Caxias Norte, 225, Campus da USP. Pirassununga, São Paulo, CEP: 13.635-350 (valinote@gmail.com).

3 Professor da Faculdade de Zootecnia e Engenharia de Alimentos da Universidade de São Paulo.

4 Doutor em Zootecnia.

${ }^{5}$ Funcionário da Faculdade de Zootecnia e Engenharia de Alimentos da Universidade de São Paulo
} 
térias Gram-positivas e aos protozoários, e aderem à partícula do alimento criando uma barreira física à ação de microrganismos e de enzimas microbianas (Jenkins, 1993).

A biohidrogenação é um mecanismo natural, realizado por microrganismos ruminais, que tem por função diminuir o efeito deletério dos lipídeos, promovendo a lise de lipídeos esterificados, com posterior hidrogenação dos ácidos graxos livres (Harfoot \& Hazlewood, 1988; Jenkins, 1993). Os principais responsáveis por esta hidrogenação são as bactérias Butyrivibrio fibrisolvens, Anaerovibrio lipolytica e Propionibacter (Bauman et al., 1999; Pariza et al., 2001).

O papel dos protozoários na biohidrogenação não é conclusivo. Bonhomme (1990) cita que os ciliados são responsáveis por 30 a 40\% da atividade lipolítica, além de participarem na hidrogenação de ácidos graxos. Williams \& Coleman (1988), Harfoot \& Hazlewood (1988) e Yokoyama \& Johnson (1993) afirmam que há evidências da participação dos protozoários na biohidrogenação de ácidos graxos.

Mesmo apresentando atividade lipolítica e de hidrogenação, os protozoários são sensíveis aos lipídeos, razão pela qual alguns óleos são utilizados para promover defaunação em ruminantes (Van Nevel \& Demeyer, 1988; Machamüller \& Kreuzer, 1999).

Ikwuegbu \& Sutton (1982), Towne et al. (1990) e Ivan et al. (2001), avaliando a inclusão de lipídeos em dietas para ruminantes, verificaram diminuição e até desaparecimento total de protozoários quando o nutriente foi adicionado, sendo os do gênero Entodinium os menos afetados.

Para reduzir o efeito negativo da gordura nos microrganismos ruminais, permitindo que o nutriente potencialize sua função, têm-se estudado várias fontes de lipídeos e seus efeitos na cinética ruminal. Algumas fontes amplamente utilizadas no Brasil são as sementes oleaginosas, como o caroço de algodão, que possuem liberação lenta de gordura, com pequeno efeito na função ruminal (Palmquist \& Jenkins, 1980; Coppock \& Wilks, 1991). Outras fontes são as gorduras protegidas, como os sais de cálcio de ácidos graxos, fontes lipídicas comerciais praticamente inertes à ação microbiana no ambiente ruminal (Palmquist \& Jenkins, 1980; Grummer, 1995; Valadares Filho \& Cabral, 2002).

O objetivo neste estudo foi avaliar a utilização de fontes de lipídeos e monensina sobre os protozoários ciliados e o $\mathrm{pH}$ no conteúdo ruminal de novilhos Nelore.

\section{Material e Métodos}

Foram utilizados quatro novilhos da raça Nelore, fistulados e canulados no rúmen, que permaneceram em baias com piso cimentado, bebedouros automáticos e cochos de alvenaria, contidos por cabresto e correntes.

Os animais receberam uma dieta total (Tabela 1), com cana-de-açúcar picada como volumoso e quatro concentrados diferentes - controle (CTRL), sal de cálcio de ácidos graxos (SC), caroço de algodão (CA), e caroço de algodão sem monensina (CASM). Para o cálculo das exigências nutricionais, foi utilizado o Cornell Net Carbohydrate and Protein System CNCPS (Fox et al., 1992).

Cada período experimental foi subdividido em um subperíodo de 20 dias de adaptação, para que o ambiente ruminal se adaptasse ao tratamento, e um dia de coleta de conteúdo ruminal.

A alimentação foi fornecida duas vezes ao dia, com matéria seca (MS) equivalente a $2 \%$ do peso vivo ou de modo que houvesse $10 \%$ de sobras, durante o período de adaptação. As sobras foram retiradas e pesadas diariamente antes da primeira alimentação, para controle e ajuste da ingestão. No período de coleta, a dieta fornecida correspondeu a $90 \%$ da ingestão média dos animais no período de adaptação, de forma que não houvesse sobras, evitando que os animais selecionassem o alimento.

As amostragens de conteúdo ruminal foram realizadas no vigésimo primeiro dia de cada período experimental, às $0,2,4,6$ e 8 horas após alimentação, com o auxílio de bomba de vácuo.

A determinação do $\mathrm{pH}$ do líquido ruminal foi feita logo após a coleta, utilizando peagâmetro de mesa calibrado com soluções tampão de pH 4,0 e pH 7,0.

Para cada horário de coleta, uma alíquota de $10 \mathrm{~mL}$ de conteúdo ruminal foi transferida para frasco de vidro com $10 \mathrm{~mL}$ de formaldeído a $37 \%$. As amostras foram homogeneizadas e mantidas em repouso até o momento das análises, de acordo com a metodologia de Dehority (1977), para determinação das curvas de aparecimento dos gêneros de ciliados em câmara de contagem de Sedgwick-Rafter com capacidade de 1 $\mathrm{mL}$ e microscópio ótico comum provido de retículo com área de $0,4362 \mathrm{~mm}^{2}$.

O delineamento experimental foi o quadrado latino (4 x 4) e os efeitos dos tratamentos e do tempo e a interação tempo x tratamento foram avaliados, adotando-se o procedimento Mixed do software SAS (2001). As características avaliadas em diferentes empos foram analisadas como medidas repetidas. 
Tabela 1 - Composição percentual e química ${ }^{1}$ das dietas em base seca

Table 1 - Ingredients and chemical composition of the diets, DM basis

\begin{tabular}{|c|c|c|c|c|}
\hline \multirow[t]{2}{*}{$\begin{array}{l}\text { Ingrediente } \\
\text { Ingredient }\end{array}$} & \multicolumn{4}{|c|}{$\begin{array}{c}\text { Tratamento }(\% \mathrm{MS})^{2} \\
\text { Treatment }(\% D M)\end{array}$} \\
\hline & CTRL & $\mathrm{SC}$ & $\mathrm{CA}$ & CASM \\
\hline $\begin{array}{l}\text { Milho grão } \\
\text { Corn }\end{array}$ & 29,36 & 26,54 & 21,18 & 21,18 \\
\hline $\begin{array}{l}\text { Polpa de citros } \\
\text { Citrus pulp }\end{array}$ & 37,19 & 33,61 & 26,82 & 26,82 \\
\hline $\begin{array}{l}\text { Farelo de soja } 49 \% \\
\text { Soybean meal }\end{array}$ & 12,50 & 14,00 & 9,00 & 9,00 \\
\hline $\begin{array}{l}\text { Uréia } \\
\text { Urea }\end{array}$ & 0,95 & 0,85 & 0,50 & 0,50 \\
\hline $\begin{array}{l}\text { Sal mineral } \\
\text { Mineral salt }\end{array}$ & 1,00 & 1,00 & 1,00 & 1,00 \\
\hline $\begin{array}{l}\text { Calcário } \\
\text { Limestone }\end{array}$ & - & - & 1,50 & 1,50 \\
\hline $\begin{array}{l}\text { Caroço de algodão } \\
\text { Whole cottonseed }\end{array}$ & - & - & 21,00 & 21,00 \\
\hline $\begin{array}{l}\text { Sal de cálcio de ácido graxo } \\
\text { Calcium salt of fatty acid }\end{array}$ & - & 5,00 & - & - \\
\hline $\begin{array}{l}\text { Monensina } \\
\text { Monensin }\end{array}$ & 0,027 & 0,027 & 0,027 & - \\
\hline $\begin{array}{l}\text { Cana-de-açúcar } \\
\text { Sugar cane }\end{array}$ & 19,000 & 19,000 & 19,000 & 19,000 \\
\hline $\begin{array}{l}\text { Total } \\
\text { Total }\end{array}$ & 100,00 & 100,00 & 100,00 & 100,00 \\
\hline
\end{tabular}

Nutriente

Nutrient

\begin{tabular}{|c|c|c|c|c|}
\hline Matéria seca & 63,25 & 63,46 & 63,56 & 63,54 \\
\hline $\begin{array}{l}\text { Dry matter } \\
\text { Proteína bruta }\end{array}$ & 16,42 & 17,02 & 15,65 & 14,81 \\
\hline $\begin{array}{l}\text { Crude protein } \\
\text { Extrato etéreo } \\
\text { Etherextract }\end{array}$ & 4,93 & 9,58 & 9,12 & 7,79 \\
\hline $\begin{array}{l}\text { Ether extract } \\
\text { Fibra detergente neutro } \\
\text { Neutral detergent fiber }\end{array}$ & 22,69 & 22 & 30,81 & 28,52 \\
\hline $\begin{array}{l}\text { Fibra detergente ácido } \\
\text { Acid detergent fiber }\end{array}$ & 22,18 & 19,65 & 25,42 & 20,85 \\
\hline
\end{tabular}

${ }^{1}$ Composição química avaliada por análise bromatológica (AOAC, 1995).

${ }^{2}$ CTRL - Tratamento controle; SC - Tratamento com sal de cálcio de ácido graxo; CA - Tratamento com caroço de algodão; CASM

- Tratamento com caroço de algodão sem monensina.

${ }^{1}$ Chemical composition (AOAC, 1995).

${ }^{2}$ CTRL - Control; CSFA - Calcium salt of fatty acid; MWCS - Monesin plus whole cottonseed; WCS - Whole cottonseed.

\section{Resultados e Discussão}

OpH ruminal não foi alterado pela adição de gordura (Tabela 2) e apresentou valores semelhantes aos mensurados por Ruy et al. (1996) e Chalupa et al. (1986).

As dietas contendo caroço de algodão (CA e CASM) resultaram em menor densidade de células do
Tabela 2 - Médias e erro-padrão do pH e do número dos protozoários ciliados $\left(\times 10^{4} / \mathrm{mL}\right)$ do conteúdo ruminal de novilhos Nelore recebendo diferentes fontes de gordura

Table 2 - Means and standard error of the $\mathrm{pH}$ and of the ciliate protozoa $\left(\times 10^{4} / \mathrm{mL}\right)$ of the ruminal content of Nellore steers fed different fatty sources

\begin{tabular}{|c|c|c|c|c|c|}
\hline & \multicolumn{4}{|c|}{$\begin{array}{c}\text { Tratamento }^{1} \\
\text { Treatment }\end{array}$} & \multirow{2}{*}{$\begin{array}{c}\text { Erro- } \\
\text { padrão } \\
\text { Standard } \\
\text { error }\end{array}$} \\
\hline & CRTL & $\mathrm{SC}$ & $\mathrm{CA}$ & CASM & \\
\hline $\mathrm{pH}$ & 6,33 & 6,32 & 6,41 & 6,50 & 0,14 \\
\hline \multicolumn{6}{|l|}{ Protozoários } \\
\hline Entodinium $^{2}$ & 52,81 & 49,71 & 6,01 & 9,22 & 3,46 \\
\hline Diplodinium & 1,51 & 1,64 & 0 & 0 & 0,54 \\
\hline Epidinium $^{2}$ & 0,78 & 1,04 & 0 & 0 & 0,55 \\
\hline Isotricha & 2,35 & 1,70 & 0,48 & 0,74 & 1,21 \\
\hline Dasytricha ${ }^{2}$ & 2,92 & 5,80 & 0 & 0 & 1,83 \\
\hline Eudiplodinium $^{2}$ & 4,23 & 6,03 & 0,68 & 0,64 & 0,84 \\
\hline Ostracodinium $^{3}$ & 0,86 & 1,04 & 0 & 0 & 2,58 \\
\hline Total $^{2}$ & 65,65 & 66,78 & 7,18 & 10,60 & 3,22 \\
\hline
\end{tabular}

${ }^{1}$ CTRL - Tratamento controle; SC - Tratamento com sal de cálcio de ácido graxo; CA - Tratamento com caroço de algodão; CASM - Tratamento com caroço de algodão sem monensina.

2 Tratamentos CRTL e SC não diferiram estatisticamente $(p<0,10)$ e foram diferentes estatisticamente dos tratamentos $C A$ e CASM.

${ }^{3}$ Interação tempo $\mathrm{x}$ tratamento.

${ }^{1}$ CTRL - Control; CSFA - Calcium salt of fatty acid; MWCS - Monesin plus whole cottonseed: WCS - Whole cottonseed.

${ }^{2}$ CRTL and CSFR treatments did not differ signficantly $(p<0.10)$, but differed from MWCS and CASM treatments.

${ }^{3}$ Time $x$ treatment interaction.

gênero Entodinium ( $<<0,01)$, enquanto os tratamentos CRTL e SC foram semelhantes para este gênero.

Os protozoários do gênero Entodinium foram encontrados em maior número absoluto para todos os tratamentos, semelhante ao observado por Towne et al. (1990), ao adicionarem gordura nas formas de sebo (4\%) ou na forma protegida $(3,5 \%)$, em dietas para bovinos de corte.

Os protozoários dos gêneros Diplodinium, Epidinium e Dasytricha apresentaram comportamento similar ( $\mathrm{p}>0,10)$, embora, nos tratamentos contendo caroço de algodão, não tenham sido encontrados protozoários dos gêneros Epidinium e Dasytricha.

Para o gênero Isotricha, não houve diferença entre os tratamentos $(p>0,10)$. Entretanto, os tratamentos com caroço de algodão apresentaram valores absolutos menores que os demais. 
Os protozoários do gênero Eudiplodinium foram encontrados em menor número nas dietas contendo caroço de algodão, que foram diferentes das demais $(\mathrm{p}<0,01)$.

Para o gênero Ostracodinium, houve interação tempo $x$ tratamento $(\mathrm{p}<0,05)$, de maneira que o número de protozoários na digesta dos animais alimentados com dieta contendo caroço de algodão foi inferior $(\mathrm{p}<0,05)$ ao das outras durante os tempos de colheita, e os tratamentos CRTL e SC não apresentaram diferença entre si nos horários de amostragem.

As dietas contendo caroço de algodão diminuíram $(\mathrm{p}<0,01)$ a quantidade total de protozoários ciliados, de modo que os animais apresentaram menor número de células ciliadas que os dos demais tratamentos $(\mathrm{p}<0,01)$, provavelmente em razão da liberação de gordura desse ingrediente, que, apesar de lenta, pode ter sido suficientemente tóxica aos ciliados. Não houve diferença estatística entre as dietas contendo caroço de algodão $(p>0,10)$ nem entre as dietas CRTL e $\mathrm{SC}(\mathrm{p}>0,10)$ para o número total de protozoários ciliados na digesta ruminal dos animais.

Para nenhum dos gêneros estudados, assim como para o total de protozoários ciliados, houve diferença estatística entre as dietas CRTL e SC, o que sugere que o sal de cálcio de ácidos graxos foi inerte no ambiente ruminal, não afetando os protozoários ciliados (Van Nevel \& Demeyer, 1988).

O efeito deletério da gordura, em relação aos protozoários ciliados neste experimento, está de acordo com a literatura (Ikwuegbu \& Sutton, 1982; Towne et al., 1990; Van Nevel \& Demeyer, 1988); Machamüller \& Kreuzer, 1999; Ivan et al., 2001), que relata toxicidade para a fauna ruminal quando gorduras insaturada e saturada com níveis de inclusão de 3,5 a 6,0\% da matéria seca foram oferecidas aos animais.

Vários autores (Van Nevel \& Demeyer, 1988; Bonhome, 1990; Chaudhary et al., 1995) relatam que há efetiva atuação dos ciliados na degradação de carboidratos estruturais, sobretudo em dietas contendo alta proporção de concentrado. Além da adesão do lipídeo à partícula do alimento e do efeito tóxico às bactérias fibrolíticas, a redução no número de protozoários pode diminuir a degradação da fibra dietética quando gordura é utilizada na dieta de ruminantes, principalmente em dietas com alto concentrado. Assim, esses tratamentos com caroço de algodão podem refletir em menor degradação da fibra.
A redução ou eliminação dos protozoários pode ainda estar associada a melhor eficiência microbiana, pelo fato de esses engolfarem bactérias, embora com possível redução na concentração de amônia ruminal e menor e mais variável pH (Ivan et al. 2001), uma vez que fagocitam partículas de amido, diminuindo a rápida fermentação do carboidrato e mantendo certa estabilidade do $\mathrm{pH}$. Os protozoários mais envolvidos na degradação de carboidratos não-estruturais e açúcares solúveis são os holotrichas, destacando-se os gêneros Dasytricha e Isotricha, que apresentaram menor número de células no líquido ruminal dos animais dos tratamentos contendo caroço de algodão.

Os protozoários do gênero Entodinium fagocitam bactérias Selenomonas ruminantium e Butyrivibrio fibrosolvens na mesma taxa ou mais rapidamente que as demais (Williams \& Coleman, 1988). Uma hipótese é que animais com menor quantidade de Entodinium possuem maior número de Butyrivibrio fibrosolvens, apresentando, conseqüentemente, maior biohidrogenação. Provavelmente, os animais recebendo os tratamentos CA e CASM apresentaram maior biohidrogenação, aumentando a quantidade de ácidos graxos saturados no rúmen e no duodeno.

A monensina não afetou a população de protozoários quando utilizado caroço de algodão na dieta, uma vez que os tratamentos contendo esse alimento não foram diferentes entre si para nenhum gênero estudado, assim como na contagem total de ciliados. A monensina pode atuar inibindo a biohidrogenação (Van Nevel \& Demeyer,1995; Fellner et al., 1997; Jenkins et al., 2003), favorecendo maior quantidade de ácidos graxos insaturados no ambiente ruminal e podendo ocasionar redução na quantidade de ciliados, ação que não foi constatada. Provavelmente, a quantidade de lipídeos tenha sido suficientemente tóxica aos microrganismos, independentemente da taxa de biohidrogenação.

Resultados contraditórios da ação da monensina sobre protozoários no rúmen são encontrados na literatura. Thivend \& Jouany (1983) evidenciaram efeito tóxico do ionóforo aos protozoários. De acordo com Dennis et al. (1986) e Arakaki et al. (2000), o fornecimento contínuo de monensina pode resultar numa seleção de população de protozoários resistentes.

Os protozoários dos gêneros Diplodinium $(\mathrm{p}<0,02)$, Epidinium $(\mathrm{p}<0,10)$ e Eudiplodinium $(\mathrm{p}<0,05)$ e o número total de protozoários $(\mathrm{p}<0,04)$ foram os únicos que sofreram influência do tempo de alimentação. 


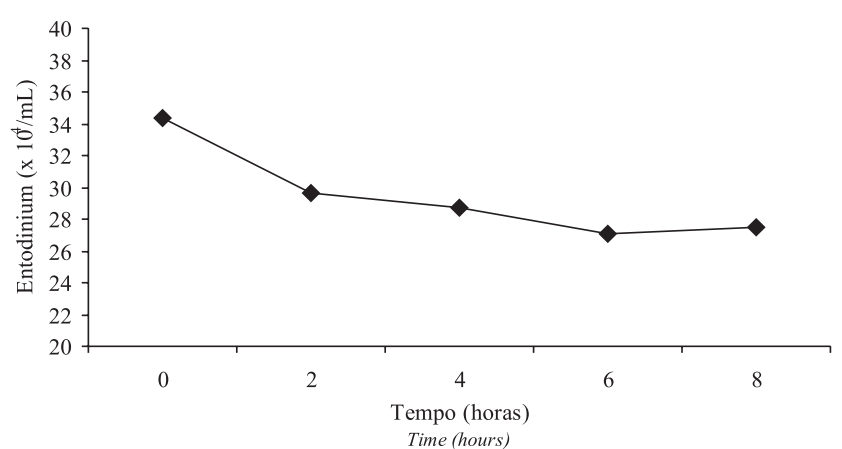

Figura 1 - Aparecimento de protozoários do gênero Entodinium ciliados, em função do tempo, no rúmen de novilhos Nelore recebendo diferentes fontes de gordura, com ou sem monensina.

Figure 1 - Effects of feeding different sources of fat, with or without monensin, on ruminal counts of entodinium after feeding in Nellore steers.

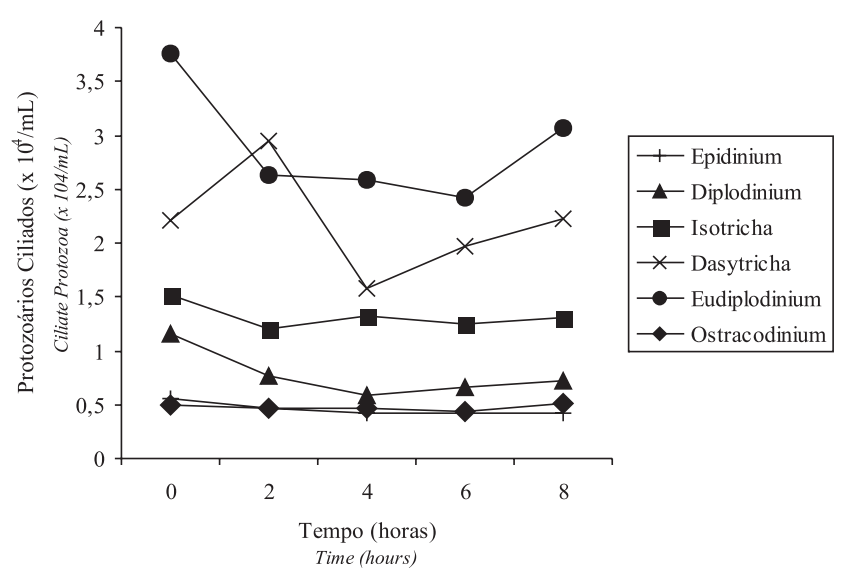

Figura 2 - Aparecimento de protozoários dos gêneros Epidinium, Diplodinium, Isotricha, Dasytricha, Eudiplodinium e Ostracodinium ciliados, em função do tempo, no rúmen de novilhos Nelore recebendo diferentes fontes de gordura, com ou sem monensina.

Figure 2 - Effects of feeding different sources of fat, with or without monensin, on ruminal counts of entodinium after feeding in Nellore steers.

Na Figura 1, está representado o número médio de protozoários do gênero Entodinium dos quatro tratamentos em função do tempo; os demais gêneros estão representados na Figura 2.

Pode-se observar, para os gêneros estudados, com exceção do Dasytricha, e para contagem total de protozoários, que, logo após a alimentação, houve redução do número de ciliados, com certa estabilização nos horários intermediários e posterior aumento do número dos microrganismos. Por se tratar de dietas com alto teor de concentrado ( $81 \%$ de concentrado na MS), a redução do $\mathrm{pH}$ pode ter sido suficiente para diminuir a quantidade de protozoários ciliados. A redução ou eliminação de protozoários ciliados pode ser um dos motivos da queda na degradação de fibra em dietas com alto nível de concentrado, o que pode ser interessante, uma vez que essas dietas possuem poucos carboidratos estruturais. Por outro lado, há possibilidade de um $\mathrm{pH}$ mais baixo e variável e de menores concentrações de amônia (Ivan et al., 2001).

\section{Conclusões}

Apenas a gordura liberada pelo caroço de algodão reduziu o número de protozoários ciliados no rúmen, enquanto os sais de cálcio de ácidos graxos foram inertes no ambiente ruminal.

A utilização de monensina em dietas contendo caroço de algodão não influenciou o aparecimento de protozoários ciliados no rúmen de bovinos Nelore.

\section{Agradecimento}

À Fundação de Amparo à Pesquisa do estado de São Paulo, pela concessão de auxílio, Processo n ${ }^{\circ}$ 02/04836-1.

\section{Literatura Citada}

ASSOCIATION OF OFFICIAL ANALYTICAL CHEMISTS AOAC. Official methods of analysis. 11.ed. Washington, D.C.: 1995. 1051p.

ARAKAKI, R.C.; STAHRINGER, R.C.; GARRET, J.E. et al. The effects of feeding monensin and yeast culture, alone or in combination, on the concentration and generic composition of rumen protozoa in steers fed on low-quality pasture supplemented with increasing levels of concentrate. Animal Feed Science and Technology, v.84, p.121-127, 2000.

BAUMAN, D.E.; BAUMGARD, L.H.; CORL, B.A. et al. Biosynthesis of conjugated linoleic acid in ruminants. Proceedings of the American Society of Animal Science, Ruminant Reseach, v.41, p.215-227, 2001. Disponível em: http://www.asas.org/symposia/proceedings. 1999.

BONHOMME, A. Rumen ciliates: their metabolism and relationships with bacteria and their hosts. Animal Feed Science and Technology, v.30, p.203-266, 1990.

CHALUPA, W.; RICKABAUGH, B.; KRONFELD, D.S. et al. Rumen fermentation in vitro as influenced by long chain fatty acids. Journal of Dairy Science, v. 67, p. 1439-1444, 1984.

CHALUPA, W.; VECCHIARELLI, B.; ELSER, A.E. et al. Ruminal fermentation in vivo as influenced by long chain fatty acids. Journal of Dairy Science, v.69, p.1293-1301, 1986. 
CHAUDHARRY, L.C.; SRIVASTAVA, A.; SINGH, K.K. Rumen fermentation pattern and digestion of structural carbohydrates in buffalo (Bubalus bubalis) calves as affected by ciliate protozoa. Animal Feed Science and Technology, v.56, p.111-117, 1995.

COPPOCK, C.E.; WILKS, D.L. Supplemental fat in high-energy rations for lactating cows: effect on intake, digestion, milk yield and composition. Journal of Animal Science, v. 69, n.9, p.3826-3837, 1991.

DEHORITY, B.A. Classification and morphology of rumen protozoa. Wooster: Ohio Agricultural Research and Development Center, 1977. 82p.

DENNIS, S.M.; NAGARAJA, T.G.; DAYTON, A.D. Effect of lasalocid, monensin and thiopeptin on rumen protozoa. Research in Veterinary Science, v.41, p.251-256, 1986.

FELLNER, V.; SAUER, F.D.; KRAMER, J.K.G. Effect of nigericin, monensin, and tetrosin on biohydrogenation in continuous flow-through ruminal fermenters. Journal Dairy Science, v.80, p.921-928, 1997.

FOX, D.G.; SNIFFEN, C.J.; O'CONNOR, J.D. A net carbohydrate and protein system for evaluating cattle diets: III. Cattle requirement and diet adequacy. Journal of Animal Science, v.70, p.3578-3596, 1992.

GRUMMER, R.R. Ruminal inertness vs digestibility of fat supplements: can there be harmony? In: CORNELL NUTRITION CONFERENCE FOR FEED MANUFACTURERS, 57., 1995. Proceedings... Ithaca: Cornell University, 1995, p.13-24.

HARFOOT, C.G.; HAZLEWOOD, G.P. Lipid metabolism in the rumen. In: HOBSON, H.D. (Ed.) The rumen microbial ecosystem. New York: Elsevier Science, 1988. p.285-322.

IKWUEGBU, O.A.; SUTTON, J.D. The effect of varying the amount of linseed oil supplementation on rumen metabolism in sheep. British Journal of Nutrition, v.48, p.365-375, 1982.

IVAN, M.; MIR, P.S.; KOENNG, K.M. et al. Effects of dietary sunflower seed oil on rumen protozoa population and tissue concentration of conjugated linoleic acid in sheep. Small Ruminant Reseach, v.41, p.215-227, 2001.

JENKINS, T.C. Lipid metabolism in the rumen. Journal of Dairy Science, v.76, p.3851-3863, 1993.

MACHAMÜLLER, A.; KREUZER, M. Methane suppresion by coconut oil and associated effects on nutrient and energy balance in sheep. Canadian Journal of Animal Science, v.79, p.65-72, 1999.

PALMQUIST, D.L.; JENKINS, T.C. Fat in lactation rations: review. Journal of Dairy Science, v.63, p.1-14, 1980.
PALMQUIST, D.L.; WEISBJERG, M.R.; HVELPLUND, T. Ruminal, intestinal, and total digestibilities of nutrients in cow fed diets high in fat and undegradable protein. Journal of Dairy Science, v.76, p.1353-1364, 1993.

PARIZA, M.W.; PARK, Y.; COOK, M.E. The biologically active isomers of conjugated linoleic acid. Progress in Lipid Research, v.40, p.283-298, 2001.

RUY, D.C.; LUCCI, C.S.; MELOTTI, L. et al. Degradação da proteína e fibra do caroço de algodão integral (Gossypium hirsutum L.) no rúmen. Brazilian Journal of Veterinary Research of Animal Science, v.33, p.276-280, 1996.

STATISTICAL ANALYSES SYSTEM - SAS. SAS user's guide: statistics. 5.ed. Cary: 2001.

THIVEND, P.; JOUANY, J.P. Effect of lasalocid sodium on rumen fermentation and digestion in sheep. Reproduction Nutrition Development, v.23, p.817-828. 1983.

TOWNE, G.; NAGARAJA, T.G.; BRANDT JR., R.T. et al. Ruminal ciliated protozoa in cattle fed finishing diets with or without supplemental fat. Journal of Animal Science, v.68, p.2150-2155, 1990 .

VALADARES FILHO, S.C.; CABRAL, S.C. Aplicação dos princípios de nutrição de ruminantes em regiões tropicais REUNIÃO DA SOCIEDADE BRASILEIRA DE ZOOTECNIA, 39., Recife. Anais... Recife: CD-ROM, 2002.

Van NEVEL, C.; DEMEYER, D.I. Lipolysis and biohydrogenation of soybean in the rumen in vitro: inhibition by antimicrobials. Journal of Dairy Science, v.78, p.2797-2806, 1995.

Van NEVEL, C.; DEMEYER, D.I. Manipulation of rumen fermentation. In: HOBSON, H.D. (Ed.) The rumen microbial ecosystem. New York: Elsevier Science, 1988. p.387-443.

Van SOEST, P.J. Nutritional ecology of the ruminant. Ithaca: Cornell University, 1994. p.35-336.

WILLIAMS, A.G.; COLEMAN, G.S. The Rumen protozoa. In: HOBSON, H.D. (Ed.) The rumen microbial ecosystem. New York: Elsevier Science, 1988. p.77-128.

YOKOYAMA, M.T.; JOHNSON, K.A. Microbiology of the rumen and intestine. In: CHURCH, D.C. (Ed.) The ruminant animal. Digestive physiology and nutrition. New York: John Wiley \& Sons, 1993. p.125-144.

Recebido em: 31/05/04

Aceito em: 22/03/05 